\title{
On the smallness of the cosmological constant in SUGRA models with Planck scale SUSY breaking and degenerate vacua
}

\author{
Colin Froggatt \\ Glasgow University \\ E-mail: Colin.Froggatteqlasqow.ac.uk \\ Roman Nevzorov*i \\ University of Adelaide \\ E-mail: roman.nevzorovdadelaide.edu.au
}

Holger Bech Nielsen

The Niels Bohr Institute

E-mail: bechanbi.dr

\section{Anthony Thomas}

University of Adelaide

E-mail: anthony.thomasdadelaide.edu.au

We argue that the exact degeneracy of vacua in $N=1$ supergravity can shed light on the smallness of the cosmological constant. The presence of such vacua, which are degenerate to very high accuracy, may also result in small values of the quartic Higgs coupling and its beta function at the Planck scale in the phase in which we live.

The European Physical Society Conference on High Energy Physics

22-29 July 2015

Vienna, Austria

* Speaker.

${ }^{\dagger}$ On leave of absence from the Theory Department, ITEP, Moscow, Russia. 


\section{Higgs mass and the multiple point principle}

The recent discovery of the Higgs boson allows us to determine quite precisely the parameters of the Higgs potential of the standard model (SM)

$$
V_{e f f}(H)=m^{2}(\phi) H^{\dagger} H+\lambda(\phi)\left(H^{\dagger} H\right)^{2} .
$$

Here $H$ is a Higgs doublet and $\phi$ is a norm of the Higgs field, i.e. $\phi^{2}=H^{\dagger} H$. The measured mass of the SM-like Higgs state is around $M_{H} \simeq 125-126 \mathrm{GeV}$, which corresponds to a rather small value of the Higgs quartic coupling at the electroweak (EW) scale, i.e. $\lambda \simeq 0.13$. Of critical importance here is the observation that this value of $\lambda$ is very close to its theoretical lower bound, coming from the vacuum stability constraint. Indeed, for sufficiently small values of the Higgs quartic coupling at the EW scale, $\lambda(\phi)$ decreases with increasing energy scale and can become negative at some intermediate scale, resulting in either instability or metastability of the physical vacuum. The extrapolation of the SM couplings up to the Planck scale, $M_{P l}$, using 3-loop renormalization group equations (RGEs) leads to (see, for example [四])

$$
\begin{aligned}
\lambda\left(M_{P l}\right)=-0.0143- & 0.0066\left(\frac{M_{t}}{\mathrm{GeV}}-173.34\right) \\
& +0.0018\left(\frac{\alpha_{3}\left(M_{Z}\right)-0.1184}{0.0007}\right)+0.0029\left(\frac{M_{H}}{\mathrm{GeV}}-125.15\right),
\end{aligned}
$$

where $M_{t}$ is the top quark mass and $\alpha_{3}\left(M_{Z}\right)$ is the value of the strong gauge coupling at the EW scale. Eq. $(\mathbb{L} 2)$ indicates that the value of $\lambda\left(M_{P l}\right)$ tends to be rather close to zero for any phenomenologically acceptable values of $M_{t}$ and $\alpha_{3}\left(M_{Z}\right)$. Moreover, $\lambda\left(M_{P l}\right)$ can be positive if the top quark is lighter than $171 \mathrm{GeV}$. This means that for these values of the top quark pole mass $\lambda(\phi)$ remains positive at any intermediate scale below $M_{P l}$, so that the physical vacuum is stable and the parameters of the SM can be extrapolated all the way up to $M_{P l}$ without any inconsistency.

For $M_{t} \simeq 171 \mathrm{GeV}$ the value of $\lambda\left(M_{P l}\right)$ vanishes. In this context it is worth noting that the computed value of the beta-function of $\lambda(\phi), \beta_{\lambda}=\frac{d \lambda(\phi)}{d \log \phi}$, also tends to be very small near the Planck scale. This implies that the Higgs effective potential of the SM (ㅍ.W) has two rings of minima in the Mexican hat with the same vacuum energy density. The radius of the little ring equals the EW vacuum expectation value (VEV) of the Higgs field, while the radius of the second ring is somewhat close to the Planck scale.

The presence of such degenerate vacua was predicted by the so-called Multiple Point Principle (MPP) [[]]-[B], according to which Nature chooses values of coupling constants such that many phases of the underlying theory should coexist. This scenario corresponds to a special (multiple) point on the phase diagram of the theory where these phases meet. The vacuum energy densities of these different phases are degenerate at the multiple point. The MPP applied to the SM implies that the Higgs effective potential has two degenerate vacua which are taken to be at the EW and Planck scales [ []]. The degeneracy of these vacua can be achieved only if

$$
\lambda\left(M_{P l}\right) \simeq 0, \quad \beta_{\lambda}\left(M_{P l}\right) \simeq 0 .
$$

It was shown that the MPP conditions ([L.3) are fulfilled when $M_{t}=173 \pm 5 \mathrm{GeV}$ and $M_{H}=135 \pm$ $9 \mathrm{GeV}$ [B]]. In previous articles the application of the MPP to the two Higgs doublet extension of the 
SM was studied [ [ $]$ ]-[目]. In particular, it was pointed out that the MPP can be used as a mechanism for the suppression of the flavour changing neutral current and CP-violation effects [ 6$]$.

The success of the MPP in predicting the Higgs mass suggests that one can also use it to provide an explanation for the small deviation of the cosmological constant from zero. This can be achieved by adapting the MPP to models based on $(N=1)$ local supersymmetry - supergravity (SUGRA),

\section{No-scale inspired SUGRA model with degenerate vacua}

The Lagrangian of $(N=1)$ supergravity is specified in terms of a real gauge-invariant Kähler function $G\left(\phi_{M}, \phi_{M}^{*}\right)$ and an analytic gauge kinetic function $f_{a}\left(\phi_{M}\right)$, which depend on the chiral superfields $\phi_{M}$. The function $f_{a}\left(\phi_{M}\right)$ determines the kinetic terms for the fields in the vector supermultiplets and the gauge coupling constants $\operatorname{Re} f_{a}\left(\phi_{M}\right)=1 / g_{a}^{2}$, where the index $a$ designates different gauge groups. The Kähler function is given by

$$
G\left(\phi_{M}, \phi_{M}^{*}\right)=K\left(\phi_{M}, \phi_{M}^{*}\right)+\ln \left|W\left(\phi_{M}\right)\right|^{2},
$$

where $K\left(\phi_{M}, \phi_{M}^{*}\right)$ and $W\left(\phi_{M}\right)$ are the Kähler potential and superpotential of the SUGRA model under consideration. Here we set $\frac{M_{P l}}{\sqrt{8 \pi}}=1$. In $(N=1)$ SUGRA models the breakdown of local SUSY takes place in a hidden sector that contains superfields $z_{i}$, which are singlets under the SM gauge group. The absolute value of the vacuum energy density at the minimum of the SUGRA scalar potential tends to be of order of $m_{3 / 2}^{2} M_{P l}^{2}$, where $m_{3 / 2}$ is a gravitino mass. Thus an enormous fine-tuning is required to keep the vacuum energy density in SUGRA models around the observed value of the cosmological constant $\rho_{\Lambda} \sim 10^{-123} M_{P l}^{4}$ [四].

The situation changes substantially in no-scale supergravity, where the invariance of the Lagrangian under imaginary translations and dilatations leads to the vanishing of the vacuum energy density. However these global symmetries also protect supersymmetry which must be broken. The breakdown of dilatation invariance does not necessarily result in a non-zero vacuum energy density [ [] . Let us consider the case when the dilatation invariance is broken in the superpotential of the hidden sector only. The simplest hidden sector of a SUGRA model of this type includes two superfields, $T$ and $z$, which transform differently under the imaginary translations $(T \rightarrow T+i \beta, z \rightarrow z)$ and dilatations $\left(T \rightarrow \alpha^{2} T, z \rightarrow \alpha z\right.$ ). If the superpotential and Kähler potential of the hidden sector of this SUGRA model are given by

$$
K(T, z)=-3 \ln \left[T+\bar{T}-|z|^{2}\right], \quad W(z)=\kappa\left(z^{3}+\mu_{0} z^{2}\right),
$$

then the tree level scalar potential of the hidden sector is positive definite

$$
V(T, z)=\frac{1}{3\left(T+\bar{T}-|z|^{2}\right)^{2}}\left|\frac{\partial W(z)}{\partial z}\right|^{2},
$$

so that the vacuum energy density vanishes near its global minima. The bilinear mass term for the superfield $z$ in the superpotential $W(z)$ violates dilatation invariance. The SUGRA scalar potential ([2.3) possesses two minima at $z=0$ and $z=-\frac{2 \mu_{0}}{3}$ that correspond to the stationary points of the hidden sector superpotential. In the first vacuum, where $z=-\frac{2 \mu_{0}}{3}$, local SUSY is broken and the 
gravitino becomes massive. Because, in general, $\mu_{0} \lesssim M_{P l}$ and $\kappa \lesssim 1$ SUSY is broken in this vacuum near the Planck scale. In the second minimum, with $z=0$, the gravitino mass vanishes and local SUSY remains intact.

The SUGRA model discussed above leads to a natural realisation of the MPP. The successful implementation of the MPP in $(N=1)$ supergravity requires the existence of a vacuum in which the low-energy limit of this theory is described by a pure supersymmetric model in flat Minkowski space. According to the MPP this vacuum and the physical one must be degenerate [ [ $]$ ] -[1]4]. Such a second vacuum is only realised if the SUGRA scalar potential has a minimum where $m_{3 / 2}=0$. This would normally requires an extra fine-tuning [9]. In the SUGRA model with the superpotential and Kähler potential (R.2) the MPP conditions are fulfilled automatically, without any extra finetuning at the tree-level. Quantum corrections to the Lagrangian of this no-scale inspired SUGRA model are expected to spoil the degeneracy of the vacua, giving rise to a huge energy density in the vacuum where SUSY is broken. Therefore such model should be considered as a toy example only. It demonstrates that, in $(N=1)$ supergravity, there might be a mechanism which ensures the vanishing of vacuum energy density in the physical vacuum. Such a mechanism can also result in a set of degenerate supersymmetric and non-supersymmetric Minkowski vacua, leading to the realization of the multiple point principle.

\section{Implications for cosmology and Higgs phenomenology}

Let us now assume that a SUGRA model with at least two vacua, which are exactly degenerate, is realised in Nature. In the first (physical) vacuum the spontaneous breakdown of SUSY takes place near the Planck scale. In the second vacuum the low-energy limit of the theory is described by a pure SUSY model in flat Minkowski space. Because the vacuum energy density of supersymmetric states in flat Minkowski space vanishes and all vacua in the MPP inspired SUGRA models are degenerate, the cosmological constant problem in the physical vacuum is solved to first approximation by our assumption. At the same time non-perturbative effects in the hidden sector can lead to the breakdown of SUSY in the supersymmetric phase at low energies, resulting in a small vacuum energy density. This small value should be then transferred to our vacuum by the assumed degeneracy.

Further, we assume that in the SUSY Minkowski vacuum vector supermultiplets associated with the unbroken gauge symmetry in the hidden sector remain massless. These vector supermultiplets may give rise to the breakdown of SUSY near the scale $\Lambda_{S Q C D}$, where the supersymmetric QCD type interactions in the hidden sector become strong in this second vacuum. The appropriate breakdown of supersymmetry can be caused by the formation of a gaugino condensate. This condensate itself does not break global SUSY. However the inclusion of non-renormalisable terms in the Lagrangian of the $(N=1)$ SUGRA model can result in a non-trivial dependence of the gauge kinetic function $f_{X}\left(z_{m}\right)$ on the hidden sector superfields $z_{m}$. As a consequence, auxiliary fields $F^{z_{m}}$ associated with superfields $z_{m}$ acquire non-zero VEVs, which are set by $<\bar{\lambda}_{a} \lambda_{a}>\simeq \Lambda_{S Q C D}^{3}$, i.e.

$$
F^{z_{m}} \propto \frac{\partial f_{X}\left(z_{k}\right)}{\partial z_{m}} \bar{\lambda}_{a} \lambda_{a} \sim \frac{\Lambda_{S Q C D}^{3}}{M_{P l}}
$$


Since the breakdown of local SUSY is caused by non-renormalisable terms that are suppressed by an inverse power of the Planck scale $M_{P l}$, the gaugino condensate gives rise to a vacuum energy density

$$
\rho_{\Lambda}^{(2)} \sim \frac{\Lambda_{S Q C D}^{6}}{M_{P l}^{2}},
$$

which is many orders of magnitude lower than $\Lambda_{S Q C D}^{4}$.

Because of the postulated exact degeneracy of vacua, the physical phase, where we live, should have the same energy density as the phase in which SUSY breaking is induced by the gaugino condensate in the hidden sector. Then from Eq. (3.2) it follows that one can reproduce the observed value of the dark energy density if $\Lambda_{S Q C D}$ is relatively close to $\Lambda_{Q C D}$ in the physical vacuum [14], i.e.

$$
\Lambda_{S Q C D} \sim \Lambda_{Q C D} / 10 .
$$

Although there is no compelling reason to expect that these two scales should be relatively close, $\Lambda_{Q C D}$ and $M_{P l}$ can be considered as the two most natural choices for the scale of dimensional transmutation in the hidden sector in the second vacuum. In the case when the QCD type interactions in the hidden sector are described by $S U$ (3) SUSY gluodynamics the corresponding value of $\Lambda_{S Q C D}$ can be obtained when the $S U(3)$ gauge coupling $g_{X}\left(M_{P l}\right) \simeq 0.654$, which is just slightly larger than the value of the QCD gauge coupling at the Planck scale in the SM, i.e. $g_{3}\left(M_{P l}\right)=0.487$.

In principle, there may also be a third phase, which has the same energy density as the first and second phases. In this third vacuum local SUSY and EW symmetry can be broken somewhere near $M_{P l}$. Since now the Higgs VEV is rather close to $M_{P l}$ one has to take into account the interaction of the Higgs and hidden sector fields. Nonetheless, such interactions can be very weak if the VEV of the Higgs field is considerably smaller than $M_{P l}$ (say $\langle H\rangle \sim M_{P l} / 10$ ) and the couplings of the SM Higgs doublet to the hidden sector fields are suppressed. Then the VEVs of the hidden sector fields in the third and physical vacua can be almost identical. As a consequence, one can expect that the gauge couplings and $\lambda\left(M_{P l}\right)$ in the first and third phases are basically the same and the absolute value of $m^{2}$ in the Higgs effective potential is much smaller than $M_{P l}^{2}$ and $\left\langle H^{\dagger} H\right\rangle$ in the third vacuum. Therefore the existence of such a third vacuum with vanishingly small energy density again implies that $\lambda\left(M_{P l}\right)$ and $\beta_{\lambda}\left(M_{P l}\right)$ are approximately zero in the third vacuum. Because the couplings in the first and third phases are basically identical, the presence of such a third vacuum leads to $\lambda\left(M_{P l}\right) \approx \beta_{\lambda}\left(M_{P l}\right) \approx 0$ in the physical vacuum.

The estimation of the dark energy density discussed here is based on the assumption that the vacua under consideration are degenerate to extremely high accuracy. In fact, the required accuracy should be of the order of the value of the cosmological constant in the physical vacuum. The desired accuracy can be achieved if the underlying theory allows only vacua which lead to a similar order of magnitude of space-time 4-volumes of the Universe at its final stage of evolution. Because the sizes of these volumes are determined by the expansion rates of the vacua associated with them, all allowed vacua should have energy densities of the same order of magnitude, i.e. of order of the observed value of the dark energy density in the phase where we live.

\section{Acknowledgments}

This work was supported by the University of Adelaide and the Australian Research Council 
through the ARC Center of Excellence in Particle Physics at the Terascale and through grant LF0 992247 (AWT). HBN thanks the Niels Bohr Institute for his emeritus status. CDF thanks Glasgow University and the Niels Bohr Institute for hospitality and support.

\section{References}

[1] D. Buttazzo, G. Degrassi, P. P. Giardino, G. F. Giudice, F. Sala, A. Salvio, A. Strumia, Investigating the near-criticality of the Higgs boson, JHEP 1312 (2013) 089 [arXiv: 1307.3536 [hep-ph] ].

[2] D. L. Bennett, H. B. Nielsen, Predictions for nonAbelian fine structure constants from multicriticality, Int. J. Mod. Phys. A 9 (1994) 5155 [hep-ph/9311321].

[3] C. D. Froggatt, H. B. Nielsen, Standard model criticality prediction: Top mass $173+-$ 5-GeV and Higgs mass 135 +- 9-GeV, Phys. Lett. B 368 (1996) 96 [hep-ph/9511371].

[4] C. D. Froggatt, L. Laperashvili, R. Nevzorov, H. B. Nielsen, M. Sher, Implementation of the multiple point principle in the two-Higgs doublet model of type II, Phys. Rev. D 73 (2006) 095005 [hep-ph/0602054].

[5] C. D. Froggatt, R. Nevzorov, H. B. Nielsen, D. Thompson, Fixed point scenario in the Two Higgs Doublet Model inspired by degenerate vacua, Phys. Lett. B 657 (2007) 95 [arXiv: 0708.2903 [hep-ph]].

[6] C. D. Froggatt, R. Nevzorov, H. B. Nielsen, D. Thompson, On the origin of approximate custodial symmetry in the Two-Higgs Doublet Model, Int. J. Mod. Phys. A 24 (2009) 5587 [arXiv:0806.3190 [hep-ph]].

[7] C.L. Bennett et al. [WMAP Collaboration], First year Wilkinson Microwave Anisotropy Probe (WMAP) observations: Preliminary maps and basic results, Astrophys. J. Suppl. 148 (2003) 1 [astro-ph/0302207].

[8] C. Froggatt, R. Nevzorov, H.B. Nielsen, On the smallness of the cosmological constant in SUGRA models, Nucl. Phys. B 743 (2006) 133 [hep-ph / 0511259 ].

[9] C. Froggatt, L. Laperashvili, R. Nevzorov, H.B. Nielsen, Cosmological constant in SUGRA models and the multiple point principle, Phys. Atom. Nucl. 67 (2004) 582 [hep-ph/ 0310127 ].

[10] C.D. Froggatt, R. Nevzorov, H.B. Nielsen, Smallness of the cosmological constant and the multiple point principle, in proceedings of 2007 Europhysics Conference on High Energy Physics (EPS-HEP 2007), J. Phys. Conf. Ser. 110 (2008) 072012 [arXiv:0708.2907 [hep-ph]].

[11] C.D. Froggatt, R. Nevzorov, H.B. Nielsen, On the Smallness of the Cosmological Constant in SUGRA Models Inspired by Degenerate Vacua, in proceedings of 17th International Conference on Supersymmetry and the Unification of Fundamental Interactions (SUSY 2009) AIP Conf. Proc. 1200 (2010) 1093 [arXiv:0909.4703 [hep-ph]].

[12] C. Froggatt, R. Nevzorov, H.B. Nielsen, Dark Energy density in Split SUSY models inspired by degenerate vacua, in proceedings of 35th International Conference on High Energy Physics (ICHEP 2010), POS (ICHEP2010) 442 (2010) [arXiv:1012.5121 [hep-ph]].

[13] C. Froggatt, R. Nevzorov, H.B. Nielsen, Dark Energy density in models with Split Supersymmetry and degenerate vacua, Int. J. Mod. Phys. A 27 (2012) 1250063 [arXiv: 1103.2146 [hep-ph]].

[14] C.D. Froggatt, R. Nevzorov, H.B. Nielsen, A.W. Thomas, Cosmological constant in SUGRA models with Planck scale SUSY breaking and degenerate vacua, Phys. Lett. B 737 (2014) 167 [arXiv:1403.1001 [hep-ph]]. 